\title{
Câncer relacionado ao trabalho: uma revisão sistemática
}

Occupational cancer: a sistematic review

\author{
Carolina Costa Chagas ${ }^{1}$ Raphael Mendonça Guimarães², Patrícia Moraes Mello Boccolini
}

\begin{abstract}
Resumo
A exposição a substâncias carcinogênicas é reconhecidamente maior nos ambientes de trabalho, e muitas destas foram assim classificadas a partir de estudos epidemiológicos realizados com populações trabalhadoras. O objetivo do estudo foi realizar uma revisão integrativa sobre os principais agentes carcinogênicos presentes no ambiente de trabalho, bem como as principais topografias acometidas por estas substâncias. Foi realizado levantamento nas bases de dados LILACS e MEDLI$\mathrm{NE}$, cujo termo de busca foi "occupational cancer" e "câncer ocupacional". Observou-se que os artigos apontam para o fato de ser provável que a exposição ambiental/ocupacional à agentes químicos de uma forma geral esteja contribuindo também para o adoecimento da população, sem que uma relação entre causa e efeito seja estabelecida. A difusão de substâncias químicas não ocorre exclusivamente através das conhecidas formas de poluição. Portanto, é de fundamental importância a construção de um sistema de vigilância destes agravos que cumpra dois objetivos principais: a identificação e controle de ambientes de trabalho onde existe potencial exposição à agentes carcinogênicos e a sistematização de informações necessárias à realização de estudos epidemiológicos de caráter exploratório ou analítico.
\end{abstract}

Palavras-chave: câncer; saúde do trabalhador; saúde pública.

\begin{abstract}
Exposure to carcinogenic substances is known in most work environments, and many of these were classified as from epidemiological studies on working populations. The objective of this study is an integrative review on the main carcinogens at work environment as well as the main topographies affected by these substances. Survey was conducted in the databases MEDLINE and LILACS, whose term has been "câncer ocupacional" and "occupational cancer". It was observed that the articles point to the fact that it is likely that exposure to environmental/occupational to chemicals in general is also contribute to the disease population, without any relationship between cause and effect is established. The diffusion of chemicals does not occur exclusively through the known forms of pollution. It is therefore of paramount importance to build a surveillance system that meets these injuries two main objectives: to identify and control work environments where there is potential exposure to carcinogenic agents and systematization of information necessary to conduct epidemiological studies, whether exploratory or analytical.
\end{abstract}

Keywords: cancer; occupational health; public health.

Trabalho realizado no Instituto de Estudos de Saúde Coletiva da Universidade Federal do Rio de Janeiro (UFRJ) - Rio de Janeiro (RJ), Brasil.

${ }^{1}$ Acadêmica de Enfermagem pela Escola de Enfermagem "Anna Nery" da UFRJ - Rio de Janeiro (RJ), Brasil.

${ }^{2}$ Professor Adjunto no Instituto de Estudos em Saúde Coletiva da UFRJ - Rio de Janeiro (RJ), Brasil.

${ }^{3}$ Cientista Social; Doutoranda no Instituto de Estudos em Saúde Coletiva da UFRJ - Rio de Janeiro (RJ), Brasil.

Endereço para correspondência: Carolina Costa Chagas - Avenida Horácio Macedo, s/n, Ilha do Fundão - CEP: 21941-598 - Rio de Janeiro (RJ), Brasil -

E-mail: carolina_riot@hotmail.com

Fonte de financiamento: nenhuma.

Conflito de interesse: nada a declarar. 


\section{INTRODUÇÃO}

Há séculos já se sabia que o trabalho, quando executado sob condições adversas, poderia causar doenças que levariam os trabalhadores à invalidez ou à morte ${ }^{1,2}$. A Organização Mundial de Saúde (OMS) define como objetivos da Saúde Ocupacional o prolongamento da expectativa de vida e a minimização da incidência de incapacidade, de doença, de dor e de desconforto; provisão de realização pessoal, fazendo com que as pessoas sejam sujeitos criativos; melhoramento da capacidade mental e física e da adaptabilidade a situações novas e mudanças das circunstâncias das situações de trabalho e de vida ${ }^{3,4}$.

O Comitê de Especialistas da OMS identifica como as "doenças relacionadas ao trabalho" de mais elevada importância, em termos de saúde pública: distúrbios comportamentais e doenças psicossomáticas; hipertensão arterial; doenças isquêmicas do coração; doenças respiratórias crônicas não específicas; doenças do aparelho locomotor; câncer e atopias (dermatites, rinites, asma brônquica) ${ }^{1}$.

Durante o século XX, inúmeras substâncias cancerígenas presentes em diferentes ambientes de trabalho foram identificadas. A International Agency for Research on Cancer (IARC) ${ }^{5}$ revisa permanentemente a literatura científica e promove inúmeros estudos a respeito da carcinogenicidade de substâncias químicas e de processos industriais, classificando-os em quatro categorias no que se refere ao potencial carcinogênico: Grupo 1, quando a substância ou mistura é carcinogênica para o homem; Grupo 2a, quando a substância ou mistura é provavelmente carcinogênica para o homem; Grupo 2b, quando a substância ou mistura é possivelmente carcinogênica para o homem; Grupo 3, quando a substância não é classificável como carcinogênica para o homem; e Grupo 4, quando a substância ou mistura provavelmente não é carcinogênica para o homem.

O câncer é uma doença multifatorial devido a um efeito combinado de fatores genéticos e externos que agem simultaneamente e seqüencialmente. A combinação de exposição ambiental com alguns polimorfismos do gene pode ser sinérgica e contribuir para uma proporção substancial do fardo do câncer na população em geral. Anualmente, cerca de 19\% de todos os cânceres são estimados para serem atribuídos ao meio ambiente, inclusive em ambientes de trabalho, resultando em 1,3 milhões de morte ${ }^{6,7}$.

De acordo com a OMS (2006), atualmente, a exposição ocupacional é a principal forma de exposição a mais de metade dos produtos químicos, grupos de produtos químicos, misturas, e exposições específicas no ambiente humano, que foram classificados pela IARC como cancerígenas para os seres humanos ${ }^{8}$. Os tumores associados ao trabalho frequentemente são observados nos órgãos em contato direto (portas de entrada e/ou eliminação) com diversos agentes carcinogênicos, por sua vez veiculados sobretudo através do ar e da dieta. São eles: pele, pulmão e trato respiratório, cavidades nasais, bexiga, rim e tubo digestivo?

A IARC classificou um total de 29 agentes e misturas relacionados ao trabalho e 12 circunstâncias de exposição como potencialmente cancerígenas aos seres humano ${ }^{6}$. No entanto, muitos outros agentes aos quais os trabalhadores estão expostos em seu ambiente de trabalho permanecem desconhecidos. A epidemiologia tem avançado no entendimento e análise das relações causais entre câncer e exposição a substâncias presentes no ambiente de trabalho, porém muitas lacunas ainda precisam ser preenchidas.

Sendo assim, o objetivo do presente artigo é sintetizar o estado da arte sobre as principais exposições ocupacionais e o risco para a ocorrência de câncer.

\section{MATERIAIS E MÉTODOS}

Trata-se de uma revisão sistemática. Foi realizada uma busca na base de referência bibliográfica MEDLINE/PubMed, tendo como chave: "occupational cancer" ou "occupation and cancer", com o corte temporal de 15 anos (1998 a 2012). Artigos originais (delineamento experimental ou observacional), cartas, artigos de revisão, meta-análise e relatórios foram considerados. Com relação ao idioma, foram selecionados artigos publicados em português, espanhol, inglês e francês, idiomas em que as revisoras independentes são fluentes e que representam significativamente trabalhos publicados em todos os continentes. A busca bibliográfica foi realizada empregando-se o programa ProCite versão 5, um gerenciador de referências bibliográficas de licença comercial que permite a eliminação de referências duplicadas e a criação de um banco de dados para o controle da distribuição das referências selecionadas.

Foi elaborado um formulário de extração de dados. Os artigos foram avaliados segundo o tema da revista, o ano de publicação, a unidade geográfica de referência (país), o tipo de publicação (artigos originais, editoriais, cartas, comentários, artigos de revisão, meta-análise, relatórios), o tipo de exposição topografia do câncer e o tipo de estudo. O processo de classificação foi realizado por dois revisores independentes em duas etapas. Na primeira, os artigos selecionados foram submetidos à leitura completa para extração dos dados. Na segunda, a resolução de discrepâncias entre revisores foi feita por consenso, com participação de um terceiro revisor independente no caso de dúvida. Foram seguidas as normas PRISMA, exceto a avaliação do risco de viés, uma vez que o objetivo era conhecer o estado arte, considerando todas as formas de exposição e topografias de câncer como desfecho. 


\section{RESULTADOS E DISCUSSÃO}

Foram recuperados, pelas chaves de busca utilizadas, 210 artigos. Após a leitura dos resumos por duas revisoras independentes, 38 foram excluídos pelo idioma (alemão, holandês, japonês, chinês, indiano, escandinavo e idioma arábico) e 63 por não tratarem diretamente de cânceres relacionados ao trabalho. Ainda, 26 foram excluídos pela não obtenção do texto completo, fosse pelo Medline ou por contato com os autores. Foram então selecionados 104 artigos para a leitura completa, também por duas revisoras independentes. Destes, 6 foram excluídos por duplicidade, restando 75 artigos para a revisão sistemática. Os estudos selecionados estão no Quadro 1.

A grande maioria dos artigos foi classificada como artigo original (78,7\%). Dentro dos estudos selecionados, 24 (32\%) são estudos de coorte, 18 (24\%) caso-controles, 1 (1,3\%) transversal, 5 (6,7\%) híbridos (caso-coorte e caso-controle aninhado a uma coorte), em um total de 48 (64\%) da evidência apresentada por estudos observacionais. Um total de 10 artigos (13,3\%) são descritivos, 1 (1,3\%) ecológico, e ainda 7 metanálises $(9,3 \%)$ e 9 revisões (12\%).
O grupo de revistas com maior representação é o de saúde ocupacional, com um total de 28 artigos (37,3\%). O grupo de periódicos classificado como saúde ambiental correspondeu a 19 artigos (25,3\%), seguido do grupo dos periódicos de clínicas, especialmente da área de oncologia, em um total de 20 artigos (26,7\%). Finalmente, o grupo de revistas classificadas como saúde pública (epidemiologia, medicina de família e afins) foi composto por 8 artigos (10,7\%). Isto reflete que as questões do câncer ocupacional, embora hegemonicamente estejam concentradas na área de saúde ambiental e saúde do trabalhador, têm se tornado foco de atenção para a clínica e para a saúde pública mais em geral.

A América do Norte lidera o número de publicações, com 29 (38,7\%) artigos. Isoladamente, os Estados Unidos são o país que mais produziram, com 25 artigos (33,3\%), sendo os outros 4 artigos $(5,3 \%)$ do Canadá. Junto com a América do Norte, a Europa publicou igualmente 29 artigos (38,7\%). Em seguida Ásia, com 9 artigos (12\%), América Latina com 5 $(6,7 \%)$ e Oceania com 3 (4\%). A África não teve nenhum artigo publicado neste sentido. Ainda, todos os artigos referentes à América Latina são do Brasil.

Quadro 1. Síntese dos estudos encontrados na revisão sistemática sobre ocupação e câncer

\begin{tabular}{|c|c|c|c|c|c|c|}
\hline Estudo & Ano & Local & Desenho & Exposição & Topografia & Periódico \\
\hline $\begin{array}{l}\text { Bosetti, Pira e } \\
\text { LaVecchia }^{12}\end{array}$ & 2005 & Itália & Revisão & Pintores & Bexiga & $\begin{array}{l}\text { Cancer Causes and } \\
\text { Control }\end{array}$ \\
\hline Band et al. ${ }^{13}$ & 2005 & Canadá & $\begin{array}{l}\text { Caso- } \\
\text { controle }\end{array}$ & $\begin{array}{l}\text { Metais, alumínio, tintas, } \\
\text { solventes, hidrocarbonetos } \\
\text { policíclicos aromáticos, óleo } \\
\text { diesel e indústria têxtil }\end{array}$ & Bexiga & $\begin{array}{l}\text { Journal of } \\
\text { Occupational \& } \\
\text { Environmental } \\
\text { Medicine }\end{array}$ \\
\hline Baena et al. ${ }^{14}$ & 2006 & Espanha & Revisão & Indústria de petróleo & Bexiga & $\begin{array}{l}\text { European Journal of } \\
\text { Cancer Prevention }\end{array}$ \\
\hline Alavanja et al. ${ }^{15}$ & 2005 & EUA & Coorte & $\begin{array}{l}\text { Cônjuge de fazendeiros, } \\
\text { comerciantes de pesticidas e } \\
\text { subsistência }\end{array}$ & $\begin{array}{l}\text { Próstata, ovário e } \\
\text { melanoma }\end{array}$ & $\begin{array}{l}\text { Scandinavian Journal } \\
\text { of Work, Environment } \\
\text { \& Health }\end{array}$ \\
\hline Lee et al. ${ }^{16}$ & 2005 & EUA & $\begin{array}{l}\text { Caso- } \\
\text { controle }\end{array}$ & Pesticidas de agricultura & Glioma & $\begin{array}{l}\text { Occupational } \\
\text { Environmental } \\
\text { Medicine } \\
\end{array}$ \\
\hline $\begin{array}{l}\text { Nichols e } \\
\text { Sorahan }^{17}\end{array}$ & 2005 & UK & Coorte & $\begin{array}{l}\text { Trabalhadores na indústria de } \\
\text { semicondutores }\end{array}$ & $\begin{array}{l}\text { Reto e melanoma - } \\
\text { homens e mulheres } \\
\text { Pâncreas - mulheres }\end{array}$ & $\begin{array}{l}\text { Occupational } \\
\text { Medicine }\end{array}$ \\
\hline Clapp $^{18}$ & 2006 & EUA & Descritivo & $\begin{array}{l}\text { Solventes e outras químicas em } \\
\text { trabalhadores da indústria de } \\
\text { computadores }\end{array}$ & $\begin{array}{l}\text { Cerebro, SNC, } \\
\text { rim, melanoma e } \\
\text { pancreas - homens } \\
\text { Rim, sistema linfático } \\
\text { e tecido sanguineo - } \\
\text { mulheres }\end{array}$ & $\begin{array}{l}\text { Environmental Health: } \\
\text { A Global Access } \\
\text { Science Source }\end{array}$ \\
\hline $\begin{array}{l}\text { Clapp, Jacobs e } \\
\text { Loechler }^{19}\end{array}$ & 2008 & EUA & Revisão & $\begin{array}{l}\text { Radiação não ionizante de } \\
\text { celulares, pesticida DDT, } \\
\text { 1-3 butadieno, poluição do } \\
\text { ar, pesticidas e solventes, } \\
\text { hidrocarbonetos aromáticos, } \\
\text { fluidos de metal e óleos minerais }\end{array}$ & $\begin{array}{l}\text { Cérebro, mamas, } \\
\text { leucemia, pulmão, } \\
\text { linfoma não-Hodgkin } \\
\text { e próstata }\end{array}$ & $\begin{array}{l}\text { Reviews } \\
\text { Environmental Health }\end{array}$ \\
\hline
\end{tabular}


Quadro 1. Continuação

\begin{tabular}{|c|c|c|c|c|c|c|}
\hline Estudo & Ano & Local & Desenho & Exposição & Topografia & Periódico \\
\hline De Roos et al. ${ }^{20}$ & 2005 & China & Caso-coorte & Trabalhadores da indústria têxtil & Colo retal & Cancer Causes Control \\
\hline Ritz et al. ${ }^{21}$ & 2006 & EUA & Coorte & $\begin{array}{c}\text { Hidrazina na industria } \\
\text { aeroespacial }\end{array}$ & Pulmão, colo retal & Epidemiology \\
\hline Samanic et al. ${ }^{22}$ & 2006 & EUA & Coorte & Aplicadores de Dicamba & Pulmão e colo retal & $\begin{array}{c}\text { Environmental Health } \\
\text { Perspectives }\end{array}$ \\
\hline Meyer et al. ${ }^{23}$ & 2011 & Brasil & $\begin{array}{l}\text { Caso- } \\
\text { controle }\end{array}$ & Agricultores & Esôfago & $\begin{array}{c}\text { International Journal } \\
\text { of Hygiene and } \\
\text { Environmental Health }\end{array}$ \\
\hline Wernli et al. ${ }^{24}$ & 2006 & China & $\begin{array}{l}\text { Caso- } \\
\text { controle } \\
\text { aninhado à } \\
\text { coorte }\end{array}$ & Trabalhadores da indústria têxtil & Esôfago e estomago & $\begin{array}{c}\text { American Journal of } \\
\text { Epidemiology }\end{array}$ \\
\hline Lynge et al..$^{25}$ & 2006 & $\begin{array}{l}\text { Países } \\
\text { Nórdicos } \\
\text { (Dinamarca, } \\
\text { Suécia, } \\
\text { Noruega e } \\
\text { Finlândia) } \\
\end{array}$ & $\begin{array}{l}\text { Caso- } \\
\text { controle } \\
\text { aninhado à } \\
\text { coorte }\end{array}$ & Tetracloroetileno & $\begin{array}{l}\text { Esôfago, cervical e } \\
\text { bexiga }\end{array}$ & $\begin{array}{c}\text { Environmental Health } \\
\text { Perspectives }\end{array}$ \\
\hline Prince et al. ${ }^{26}$ & 2006 & EUA & Coorte & PCB na indústria elétrica & $\begin{array}{c}\text { Ductos biliares, } \\
\text { vesícula, fígado. } \\
\text { Intestino e sistema } \\
\text { nervoso }\end{array}$ & $\begin{array}{l}\text { Environmental Health: } \\
\text { A Global Access } \\
\text { Science Source }\end{array}$ \\
\hline Yang 27 & 2011 & Coréia do Sul & Revisão & $\begin{array}{l}\text { Amianto, sílica, pó de madeira, } \\
\text { fuligem, hidrocarbonetos } \\
\text { policíclicos aromáticos, metais } \\
\text { pesados, aminas aromáticas, } \\
\text { solventes orgânicos, radiação, } \\
\text { radônio e poluentes domésticos }\end{array}$ & $\begin{array}{l}\text { Pele, pulmão, bexiga, } \\
\text { mesotelioma e } \\
\text { leucemia }\end{array}$ & $\begin{array}{l}\text { J Environ Sci Health } \\
\text { C Environ Carcinog } \\
\text { Ecotoxicol Rev }\end{array}$ \\
\hline Hsieh et al. ${ }^{28}$ & 2011 & Taiwan & Coorte & Cloreto de polivinila & Fígado e leucemia & $\begin{array}{c}\text { Occupational } \\
\text { Environmental } \\
\text { Medicine } \\
\end{array}$ \\
\hline $\begin{array}{l}\text { Fedeli e } \\
\text { Mastrangelo }^{29}\end{array}$ & 2011 & Itália & Descritivo & Cloreto de vinila & $\begin{array}{c}\text { Carcinoma } \\
\text { hepatocelular }\end{array}$ & $\begin{array}{l}\text { American Journal of } \\
\text { Industrial Medicine }\end{array}$ \\
\hline $\begin{array}{l}\text { Ramroth H } \\
\text { et al. }{ }^{30}\end{array}$ & 2008 & Alemanha & $\begin{array}{l}\text { Caso- } \\
\text { controle }\end{array}$ & Pó de madeira & Laringe & $\begin{array}{l}\text { American Journal of } \\
\text { Industrial Medicine }\end{array}$ \\
\hline $\begin{array}{l}\text { Paget-Bailly, } \\
\text { Cyr e Luce }^{31}\end{array}$ & 2012 & França & Meta-análise & $\begin{array}{l}\text { Hidrocarbonetos aromáticos } \\
\text { policíclicos, motor de escape, pó } \\
\text { de tecido e indústria da borracha }\end{array}$ & Laringe & $\begin{array}{c}\text { Journal of } \\
\text { Occupational \& } \\
\text { Environmental } \\
\text { Medicine }\end{array}$ \\
\hline Pira et al. ${ }^{32}$ & 2009 & Itália & Coorte & Amianto crisotila & $\begin{array}{l}\text { Pleura, peritôneo, } \\
\text { pulmão e laringe }\end{array}$ & $\begin{array}{l}\text { Occupational } \\
\text { Environmental } \\
\text { Medicine }\end{array}$ \\
\hline Rushton et al. ${ }^{33}$ & 2010 & UK & Revisão & $\begin{array}{l}\text { Mesotelioma, sinusal, pulmão, } \\
\text { nasofaringe, mama, pele não } \\
\text { melanoma, bexiga, esôfago, } \\
\text { partes moles, laringe e estômago }\end{array}$ & $\begin{array}{l}\text { Asbesto, trabalho } \\
\text { em turnos, óleos e } \\
\text { solventes, radiação } \\
\text { solar, sílica, dioxinas, } \\
\text { tabagismo passivo } \\
\text { em ambiente de } \\
\text { trabalho, radônio, } \\
\text { tetracloroetileno e } \\
\text { arsênio }\end{array}$ & $\begin{array}{l}\text { British Journal of } \\
\text { Cancer }\end{array}$ \\
\hline Ramroth et al. ${ }^{34}$ & 2011 & Alemanha & $\begin{array}{c}\text { Caso- } \\
\text { controle }\end{array}$ & Amianto & Laringe & $\begin{array}{l}\text { American Journal of } \\
\text { Industrial Medicine }\end{array}$ \\
\hline Purdue et al. ${ }^{35}$ & 2006 & Suécia & Transversal & $\begin{array}{l}\text { Indústria da construção (Óleos, } \\
\text { asbestos, solvents orgânicos, } \\
\text { peira metálica, asfalto, serragem, } \\
\text { partículas rochosas, cimento) }\end{array}$ & Cabeça e Pescoço & $\begin{array}{l}\text { Scandinavian Journal } \\
\text { of Work, Environment } \\
\text { and Health }\end{array}$ \\
\hline Bonner et al. ${ }^{36}$ & 2005 & EUA & Coorte & Carbamato (inseticida) & Pulmão & $\begin{array}{c}\text { Environmental Health } \\
\text { Perspectives }\end{array}$ \\
\hline
\end{tabular}


Quadro 1. Continuação

\begin{tabular}{|c|c|c|c|c|c|c|}
\hline Estudo & Ano & Local & Desenho & Exposição & Topografia & Periódico \\
\hline Beveridge et al. ${ }^{37}$ & 2010 & Canadá & $\begin{array}{l}\text { Caso- } \\
\text { controle }\end{array}$ & Níquel, cromo VI e cádmio & Pulmão & $\begin{array}{l}\text { American Journal of } \\
\text { Industrial Medicine }\end{array}$ \\
\hline $\begin{array}{l}\text { Schubauer- } \\
\text { Berigan et al. }\end{array}$ & 2011 & EUA & Coorte & Berílio & Pulmão & $\begin{array}{l}\text { Occupational } \\
\text { Environmental } \\
\text { Medicine }\end{array}$ \\
\hline $\begin{array}{l}\text { Lehman e } \\
\text { Hein }^{39}\end{array}$ & 2006 & EUA & Descritivo & Tolueno em fábrica de calçados & Pulmão & $\begin{array}{l}\text { American Journal of } \\
\text { Industrial Medicine }\end{array}$ \\
\hline Azari et al. ${ }^{40}$ & 2010 & Irã & Descritivo & Fibra de amianto & Pulmão & Industrial Health \\
\hline $\begin{array}{l}\text { Algranti, } \\
\text { Buschinelli e } \\
\text { De Capitani }^{41}\end{array}$ & 2010 & Brasil & Revisão & $\begin{array}{l}\text { Solda, Metais, poeira } \\
\text { inorgânica, amianto e categorias } \\
\text { profissionais relacionadas }\end{array}$ & Pulmão & $\begin{array}{l}\text { Jornal Brasileiro de } \\
\text { Pneumologia }\end{array}$ \\
\hline Loomis et al. ${ }^{42}$ & 2010 & EUA & Coorte & Fibra de amianto & Pulmão & $\begin{array}{l}\text { Occupational } \\
\text { Environmental } \\
\text { Medicine }\end{array}$ \\
\hline Zhao et al. ${ }^{43}$ & 2005 & EUA & Coorte & Tricloroetileno e óleo mineral & $\begin{array}{l}\text { Bexiga, rim, pulmão, } \\
\text { melanoma, esôfago, } \\
\text { estômago, linfoma } \\
\text { não-hodgkin e } \\
\text { leucemia }\end{array}$ & $\begin{array}{l}\text { American Journal of } \\
\text { Industrial Medicine }\end{array}$ \\
\hline Christian et al. ${ }^{44}$ & 2011 & EUA & Ecológico & Mineração de carvão & Pulmão & Public Health Reports \\
\hline Hosgood et al. ${ }^{45}$ & 2012 & China & $\begin{array}{l}\text { Caso- } \\
\text { controle }\end{array}$ & Mineração de carvão & Pulmão & $\begin{array}{l}\text { American Journal of } \\
\text { Industrial Medicine }\end{array}$ \\
\hline Rage et al. ${ }^{46}$ & 2012 & França & Coorte & Mineração de urânio & Pulmão & Radiation Research \\
\hline Vacquier et al. ${ }^{47}$ & 2011 & França & Coorte & Mineração de urânio & Pulmão & Radiation Research \\
\hline $\begin{array}{l}\text { Sethi, } \\
\text { El-Ghamry e } \\
\text { Kloecker }^{48}\end{array}$ & 2012 & EUA & Descritivo & Radônio & Pulmão & $\begin{array}{l}\text { Clinical Advances } \\
\text { Hematology and } \\
\text { Oncology }\end{array}$ \\
\hline Gennaro et al. ${ }^{49}$ & 2008 & Itália & Coorte & Cloreto de vinila e polivinila & $\begin{array}{c}\text { Fígado, } \\
\text { angiossarcoma, } \\
\text { sistema hemo- } \\
\text { linfopoiético, } \\
\text { leucemia, linfoma e } \\
\text { pulmão }\end{array}$ & BMC Public Health \\
\hline $\begin{array}{l}\text { Luce, Stücker } \\
\text { e ICARE Study } \\
\text { Group }^{50}\end{array}$ & 2011 & França & $\begin{array}{l}\text { Caso- } \\
\text { controle }\end{array}$ & $\begin{array}{c}\text { Amianto, fibras minerais } \\
\text { sintéticas, formaldeído, } \\
\text { hidrocarbonetos policíclicos } \\
\text { aromáticos, compostos de cromo } \\
\text { e níquel, arsênio, pó de madeira, } \\
\text { pó têxtil, solventes, ácidos fortes, } \\
\text { fluidos de corte, sílica, fumaça } \\
\text { de óleo diesel e fumaça de } \\
\text { soldagem }\end{array}$ & $\begin{array}{c}\text { Cabeça, pescoço e } \\
\text { pulmão }\end{array}$ & BMC Public Health \\
\hline Carneiro et al. ${ }^{51}$ & 2002 & Brasil & Descritivo & Sílica & Pulmão & Jornal de Pneumologia \\
\hline Vida et al. ${ }^{52}$ & 2010 & Canadá & $\begin{array}{l}\text { Caso- } \\
\text { controle }\end{array}$ & Sílica & Pulmão & $\begin{array}{c}\text { Cancer Epidemiology, } \\
\text { Biomarkers and } \\
\text { Prevention }\end{array}$ \\
\hline Consonni et al. ${ }^{53}$ & 2012 & Itália & $\begin{array}{l}\text { Caso- } \\
\text { controle }\end{array}$ & Pedreiros & Pulmão & $\begin{array}{l}\text { American Journal of } \\
\text { Industrial Medicine }\end{array}$ \\
\hline $\begin{array}{l}\text { Wünsch-Filho } \\
\text { et al. }{ }^{54}\end{array}$ & 1998 & Brasil & $\begin{array}{l}\text { Caso- } \\
\text { controle }\end{array}$ & $\begin{array}{l}\text { Indústria de máquinas, } \\
\text { cerâmica, e têxtil }\end{array}$ & Pulmão & $\begin{array}{c}\text { Scandinavian Journal } \\
\text { of Work, Environment } \\
\text { \& Health }\end{array}$ \\
\hline Li et al. ${ }^{55}$ & 2012 & China & Coorte & Amianto e Radônio interior & $\begin{array}{c}\text { Mesotelioma, } \\
\text { leucemia, bexiga e } \\
\text { pulmão }\end{array}$ & $\begin{array}{l}\text { Occupational } \\
\text { Medicine }\end{array}$ \\
\hline Siesling et al. ${ }^{56}$ & 2012 & Europa & Descritivo & Amianto & $\begin{array}{l}\text { Traquéia, timo } \\
\text { e mesotelioma } \\
\text { peritoneal. }\end{array}$ & $\begin{array}{l}\text { European Journal of } \\
\text { Cancer }\end{array}$ \\
\hline Alder et al. ${ }^{57}$ & 2006 & UK & Meta-análise & Indústria da borracha & Leucemia & $\begin{array}{l}\text { American Journal of } \\
\text { Epidemiology }\end{array}$ \\
\hline
\end{tabular}


Quadro 1. Continuação

\begin{tabular}{|c|c|c|c|c|c|c|}
\hline Estudo & Ano & Local & Desenho & Exposição & Topografia & Periódico \\
\hline $\begin{array}{l}\text { Sorahan, } \\
\text { Kinlen e Doll }{ }^{58}\end{array}$ & 2005 & UK & Coorte & Benzeno & $\begin{array}{l}\text { Lábios, pulmões e } \\
\text { brônquios }\end{array}$ & $\begin{array}{l}\text { Occupational } \\
\text { Environmental } \\
\text { Medicine }\end{array}$ \\
\hline Glass et al. ${ }^{59}$ & 2006 & Austrália & $\begin{array}{l}\text { Caso- } \\
\text { controle } \\
\text { aninhado à } \\
\text { coorte }\end{array}$ & Benzeno & Leucemia & $\begin{array}{l}\text { Annals of the New } \\
\text { York Academy of } \\
\text { Sciences }\end{array}$ \\
\hline Khalade et al. ${ }^{60}$ & 2010 & UK & Meta-análise & Benzeno & Leucemia & $\begin{array}{c}\text { Environmental Health: } \\
\text { A Global Access } \\
\text { Science Source }\end{array}$ \\
\hline $\begin{array}{l}\text { Mills, Yang e } \\
\text { Riordan }^{61}\end{array}$ & 2005 & EUA & $\begin{array}{l}\text { Caso- } \\
\text { controle } \\
\text { aninhado à } \\
\text { coorte }\end{array}$ & Agricultores & Linfo-hematopoiético & $\begin{array}{l}\text { Cancer Causes and } \\
\text { Control }\end{array}$ \\
\hline Rafnsson $^{62}$ & 2006 & Islândia & $\begin{array}{l}\text { Caso- } \\
\text { controle } \\
\text { aninhado à } \\
\text { coorte }\end{array}$ & Hexaclorociclohexano & $\begin{array}{l}\text { Linfoma não- } \\
\text { hodgkin }\end{array}$ & $\begin{array}{l}\text { European Journal of } \\
\text { Cancer }\end{array}$ \\
\hline Lynch et al. ${ }^{63}$ & 2006 & EUA & Coorte & Aplicadores de Cianazina & $\begin{array}{c}\text { Próstata, linfo- } \\
\text { hematopoiético, } \\
\text { linfoma non-hodgkin } \\
\text { e pulmão }\end{array}$ & $\begin{array}{l}\text { Environmental Health } \\
\text { Perspectives }\end{array}$ \\
\hline Burns et al. ${ }^{64}$ & 2011 & EUA & Coorte & Ácido 2,4-diclorofenoxiacético & $\begin{array}{l}\text { Próstata e sistema } \\
\text { respiratório }\end{array}$ & $\begin{array}{c}\text { International Journal } \\
\text { of Environmental } \\
\text { Research and Public } \\
\text { Health }\end{array}$ \\
\hline Fritschi et al. ${ }^{65}$ & 2005 & Austrália & $\begin{array}{l}\text { Caso- } \\
\text { controle }\end{array}$ & $\begin{array}{l}\text { Solventes, metais, poeiras } \\
\text { orgânicas e bifenilos } \\
\text { policlorados (PCBs) }\end{array}$ & $\begin{array}{l}\text { Linfoma non- } \\
\text { Hodgkin }\end{array}$ & $\begin{array}{l}\text { Cancer Causes and } \\
\text { Control }\end{array}$ \\
\hline Purdue et al. ${ }^{66}$ & 2011 & EUA & $\begin{array}{l}\text { Caso- } \\
\text { controle }\end{array}$ & Tricloroetileno & $\begin{array}{l}\text { Linfoma non- } \\
\text { Hodgkin }\end{array}$ & $\begin{array}{c}\text { Environmental Health } \\
\text { Perspectives }\end{array}$ \\
\hline Mester et al. ${ }^{67}$ & 2006 & Alemanha & $\begin{array}{l}\text { Caso- } \\
\text { controle }\end{array}$ & Borracha e produtos de plástico & Linfoma malígno & $\begin{array}{c}\text { Occupational } \\
\text { Environmental } \\
\text { Medicine }\end{array}$ \\
\hline Pukkala et at. ${ }^{68}$ & 2009 & $\begin{array}{c}\text { Países } \\
\text { Nórdicos }\end{array}$ & Descritivo & Categorias Ocupacionais & Câncer em Geral & Acta Oncologica \\
\hline $\begin{array}{l}\text { Parkin, Mesher } \\
\text { e Sasieni69 }\end{array}$ & 2011 & UK & Descritivo & Radiação ultravioleta & Melanoma & $\begin{array}{c}\text { British Journal of } \\
\text { Cancer }\end{array}$ \\
\hline $\begin{array}{l}\text { Melkonian } \\
\text { et al. }{ }^{71}\end{array}$ & 2011 & EUA & Coorte & $\begin{array}{l}\text { Arsênio, fumo, exposição ao sol, } \\
\text { fertilizantes e pesticidas }\end{array}$ & Pele & $\begin{array}{l}\text { American Journal of } \\
\text { Epidemiology }\end{array}$ \\
\hline Bates $^{72}$ & 2007 & EUA & $\begin{array}{l}\text { Caso- } \\
\text { controle }\end{array}$ & Bombeiros & $\begin{array}{c}\text { Testículo, melanoma, } \\
\text { cérebro, esôfago e } \\
\text { próstata }\end{array}$ & $\begin{array}{l}\text { American Journal of } \\
\text { Industrial Medicine }\end{array}$ \\
\hline $\begin{array}{l}\text { Tokumaru } \\
\text { et al. }{ }^{73}\end{array}$ & 2006 & Japão & Meta-análise & Comissárias de voo & Mama e melanoma & $\begin{array}{c}\text { Journal of Travel } \\
\text { Medicine }\end{array}$ \\
\hline $\begin{array}{l}\text { Perez-Gomez } \\
\text { et al. }{ }^{74}\end{array}$ & 2005 & Suécia & Coorte & Arsênio e mercúrio & Melanoma & $\begin{array}{l}\text { American Journal of } \\
\text { Industrial Medicine }\end{array}$ \\
\hline $\begin{array}{l}\text { Bunderson- } \\
\text { Schelvan et al. }\end{array}$ & 2011 & EUA & Revisão & Amianto & Peritônio e ovários & $\begin{array}{c}\text { Journal of Toxicology } \\
\text { and Environmental } \\
\text { Health }\end{array}$ \\
\hline $\begin{array}{l}\text { Santibañez } \\
\text { et al. } .^{76}\end{array}$ & 2012 & Espanha & $\begin{array}{l}\text { Caso- } \\
\text { controle }\end{array}$ & $\begin{array}{l}\text { Pesticidas, amianto e pó de } \\
\text { madeira }\end{array}$ & Estômago & $\begin{array}{l}\text { Occupational } \\
\text { Environmental } \\
\text { Medicine }\end{array}$ \\
\hline $\begin{array}{l}\text { Chen e } \\
\text { Seaton }^{71}\end{array}$ & 1998 & UK & Meta-análise & Pintores & $\begin{array}{l}\text { Leucemia, fígado, } \\
\text { esôfago, estômago, } \\
\text { bexiga e pulmão }\end{array}$ & $\begin{array}{c}\text { Cancer Detection and } \\
\text { Prevention }\end{array}$ \\
\hline
\end{tabular}


Quadro 1. Continuação

\begin{tabular}{|c|c|c|c|c|c|c|}
\hline Estudo & Ano & Local & Desenho & Exposição & Topografia & Periódico \\
\hline $\begin{array}{l}\text { Blair e } \\
\text { Freeman }^{78}\end{array}$ & 2009 & EUA & Revisão & Agricultores & $\begin{array}{l}\text { Tecido conjuntivo, } \\
\text { linfoma não- } \\
\text { Hodgkin, pele, } \\
\text { estômago e cérebro, } \\
\text { esôfago, cólon, } \\
\text { bexiga, pulmão }\end{array}$ & $\begin{array}{c}\text { Journal of } \\
\text { Agromedicine }\end{array}$ \\
\hline $\begin{array}{l}\text { Arias Bahia, } \\
\text { Echenique } \\
\text { Mattos e } \\
\text { Koifman }^{79}\end{array}$ & 2005 & Brasil & Descritivo & Pó de madeira & $\begin{array}{l}\text { Cavidade oral/faringe, } \\
\text { estômago e fígado }\end{array}$ & $\begin{array}{l}\text { Environmental } \\
\text { Research }\end{array}$ \\
\hline $\begin{array}{l}\text { Lie, Andersen } \\
\text { e Kjaerheim }{ }^{80}\end{array}$ & 2007 & Noruega & Coorte & Enfermeiras & $\begin{array}{l}\text { Mama, ovário, } \\
\text { melanoma e pele }\end{array}$ & $\begin{array}{c}\text { Scandinavian Journal } \\
\text { of Work, Environment } \\
\text { and Health }\end{array}$ \\
\hline Camargo et al. ${ }^{81}$ & 2011 & EUA & Meta-análise & Amianto & Ovário & $\begin{array}{c}\text { Environmental Health } \\
\text { Perspectives }\end{array}$ \\
\hline $\begin{array}{l}\text { Reid, Klerk e } \\
\text { Musk }^{82}\end{array}$ & 2011 & Austrália & Meta-análise & Amianto & Ovário & $\begin{array}{c}\text { Cancer Epidemiology, } \\
\text { Biomarkers and } \\
\text { Prevention }\end{array}$ \\
\hline Mahajan et al. ${ }^{83}$ & 2006 & EUA & Coorte & Fonofós (Organofosforado) & Próstata & $\begin{array}{c}\text { Environmental Health } \\
\text { Perspectives }\end{array}$ \\
\hline Mahajan et al. ${ }^{84}$ & 2006 & EUA & Coorte & Forato (Organofosforado) & Próstata & $\begin{array}{c}\text { Environmental Health } \\
\text { Perspectives }\end{array}$ \\
\hline Bassil et al. ${ }^{85}$ & 2007 & Canadá & Revisão & Pesticidas & $\begin{array}{l}\text { Linfoma não } \\
\text { Hodgking, leucemia, } \\
\text { cérebro, próstata, } \\
\text { Rim - crianças }\end{array}$ & $\begin{array}{l}\text { Canadian Family } \\
\text { Physician }\end{array}$ \\
\hline Prince et al. ${ }^{86}$ & 2006 & EUA & Coorte & Bifenilos policlorados (PCBs) & $\begin{array}{c}\text { Fígado } \\
\text { Estômago e próstata - } \\
\text { homens } \\
\text { Intestino - mulheres }\end{array}$ & $\begin{array}{c}\text { Environmental Health } \\
\text { Perspectives }\end{array}$ \\
\hline Vinceti et al. ${ }^{87}$ & 2007 & Itália & $\begin{array}{c}\text { Caso- } \\
\text { controle }\end{array}$ & Cádmio & Próstata & $\begin{array}{l}\text { The Science of the } \\
\text { Total Environment }\end{array}$ \\
\hline
\end{tabular}

Conforme esperado, a produção se concentra em dois pilares: os estudos que focaram especificamente em substâncias químicas, em um total de 53 (70,7\%); e aqueles cujo foco foi as categorias ocupacionais $(29,3 \%)$. Dentro do grupo de substâncias químicas, 19 (25,3\%) avaliaram metais pesados e poeiras inorgânicas, 15 (20\%) avaliaram solventes e 18 (24\%) avaliaram agrotóxicos.

Finalmente, há 47 diferentes topografias descritas nos estudos. Entre eles, as 10 que mais se destacam são: pulmão (38,7\%); próstata (13,3\%); leucemia (14,7\%); bexiga, cérebro e melanoma (12\%); linfoma (10,7\%); fígado e estômago (9,3\%); e esôfago (8\%).

Pode-se dividir, para efeito de análise, os artigos referentes ao risco atribuível e à força de associação.

\section{Risco Atribuível}

Numa publicação da OMS (2006) encontramos a relação entre o percentual do risco atribuível à população para a mortalidade por câncer e as principais exposições carcinogênicas decorrentes do ambiente de trabalho. Com isso, a seguir, foi possível obter um breve panorama acerca dos recentes estudos que tem sido publicados referentes ao tema ${ }^{8}$.

Para o câncer de pulmão, Steenland et al. ${ }^{10}$ encontram um risco atribuível entre 6,3 e 13\%, enquanto Nurminen e Karjalainen ${ }^{11}$ revelam $24 \%$ em seu estudo. Como exemplo de principais exposições carcinogênicas ocupacionais temos o amianto, a sílica, o níquel, o radão interior, a fumaça de óleo diesel, a fumaça do tabaco proveniente do meio ambiente no ambiente de trabalho, a produção e refino do arsênico, o berílio, o cádmio, o alumínio, o cromo, a mineração de urânio, a fundição de cobre, aço e ferro, e trabalhadores de vinha, telheiros, trabalhadores de asfalto e pintores ${ }^{8}$.

De acordo com Steenland et al. ${ }^{10}$, em torno de 7 a $19 \%$ da população possui um risco atribuível para a mortalidade por câncer de bexiga, no entanto, Nurminen e Karjalainen ${ }^{11}$ estimam um percentual de 10,3 de risco para a população relacionado a mesmo câncer. Exposições ocupacionais a substâncias como a 2-naftilamina, benzidina e 4-aminobifenilo 
são consideradas carcinogênicas. Os indivíduos participantes na fabricação de magenta, auramina, p-cloro-o-toluidina, pigmento de cromato e corantes, produção de látex sintético, vulcanização de pneus, recicladores, fabricantes de cabos e os trabalhadores de usinas de gás também estão expostos à carcinogenicidade das substâncias envolvidas no trabalho.

Foi encontrado um risco atribuível entre 85 e $90 \%$ para o mesotelioma, de acordo com Steenland et al. ${ }^{10}$, e 71,3\%, segundo Nurminen e Karjalainen ${ }^{11}$. A exposição ao amianto se caracteriza como o principal carcinogênico deste caso.

Para a leucemia, o risco atribuível encontrado por Steenland et al. ${ }^{10}$ estabeleceu-se entre 0,8 e 2,8\%, e, de acordo com Nurminen e Karjalainen ${ }^{11}$, este risco sobe para $10,9 \%$ da população. Os principais agentes carcinogênicos ocupacionais para a leucemia são a radiação ionizante externa, o benzeno, o óxido de etileno, a indústria da borracha, a fabricação e o reparo de botas e sapatos.

Steenland et al. ${ }^{10}$ relataram que o risco atribuível para o câncer de laringe encontra-se entre 1,5 e 20\%. As principais substâncias carcinogênicas ocupacionais são o ácido sulfúrico, óleos minerais e o amianto, além das operações de decapagem que também se constituem como exposição de potencial carcinogênico.

O câncer de pele possui, nos estudos de Steenland et al. ${ }^{10}$, um risco atribuível de 1,5 a $6,0 \%$. Como exemplo das principais exposições carcinogênicas do ambiente de trabalho, encontramos a radiação solar intensiva, os arremessos de alcatrão de carvão, o alcatrão de carvão, os óleos de xisto, o arsênico, os óleos minerais, os hidrocarbonetos aromáticos policíclicos. As exposições a que se submetem os trabalhadores da produção de coque, trabalhadores de vinha e pescadores também integram a tabela de carcinogenicidade.

Para o câncer de nasofaringe e sinusal foi encontrado um risco atribuível que varia entre 33 e $46 \%$, segundo o estudo de Steenland et al. ${ }^{10}$. Compostos e substâncias como pó de madeira, compostos de níquel, cromo hexavalente e formaldeído, assim como atividades tais quais a fabricação e reparo de botas e sapatos, a fabricação de isopropanol utilizando o processo de ácido forte, a fabricação de móveis, marcenaria e carpintaria, constituem as principais exposições carcinogênicas ocupacionais.

A porcentagem encontrada por Steenland et al. ${ }^{10} \mathrm{em}$ relação ao risco atribuível para o câncer de rim foi de 0 a 2,3\% da população. A principal atividade laboral com alta exposição carcinogênica para este caso é a produção de coque.

Nos casos de câncer de fígado, o risco atribuível foi estabelecido entre 0,4 e 1,1\%, de acordo com Steeland et al. ${ }^{10}$. As principais exposições carcinogênicas ocupacionais são ao cloreto de vinila, às infecções ocupacionais com hepatite B e $\mathrm{C}$ e aos trabalhadores da área de saúde.

\section{Estudos de Associação}

\section{Câncer de Bexiga}

Um estudo do tipo caso-controle de base populacional realizado no Canadá observou uma associação positiva entre câncer de bexiga e exposição ocupacional a tintas em trabalhadores da indústria têxtil ${ }^{12}$ e outro estudo avaliou a associação desse sítio de câncer e trabalhadores da indústria do alumínio ${ }^{13}$. Exposição a hidrocarbonetos aromáticos policíclicos e diesel proveniente de emissões de motores também foi associado a um risco aumentado para o desenvolvimento de câncer de bexiga ${ }^{14}$.

\section{Câncer de Cérebro}

Estudos recentes têm encontrado evidências de associação entre câncer de cérebro e Sistema Nervoso Central (SNC) e exposição a agrotóxicos. Um estudo do tipo coorte realizado nos EUA, o Agricultural Health Study, observou um aumento no risco de câncer de cérebro e SNC entre aplicadores de agrotóxicos ${ }^{15}$. Outro estudo realizado nos EUA também observou um risco aumentado de câncer de cérebro e SNC em trabalhadores expostos à tinta de cabelo ${ }^{16}$. Alguns estudos também tem apontado para um aumento no risco de câncer de cérebro e SNC em trabalhadores envolvidos na fabricação de computadores e semicondutores ${ }^{17,18}$.

\section{Câncer de Cólon}

Poucos estudos identificaram uma associação entre ocupação e câncer de cólon ${ }^{19}$. Um estudo do tipo caso-controle aninhado realizado na China verificou um aumento no risco de câncer de cólon em trabalhadoras da indústria têxtil expostos por um longo período (20 anos ou mais) a corantes ${ }^{20}$. Outro estudo encontrou um aumento no risco de câncer de cólon em trabalhadores aeroespaciais expostos à hidrazina ${ }^{21}$. Por fim, um recente achado da Agricultural Health Study observou um risco aumentado de câncer de cólon entre aplicadores de agrotóxicos expostos ao herbicida dicamba ${ }^{22}$.

\section{Câncer de Esôfago}

Trabalhadores agrícolas apresentaram maior risco de desenvolver câncer de esôfago quando comparados a trabalhadores não agrícolas em um estudo do tipo caso-controle, com base em certificados de óbitos, realizado na região Sul do Brasil ${ }^{23}$. Outros estudos também encontraram associação entre câncer de esôfago e trabalhadores da indústria têxti ${ }^{24}$, 
bem como trabalhadores de tinturarias e de estabelecimentos que fazem limpeza a seco expostos ao tetracloroetileno ${ }^{25}$.

\section{Câncer de Fígado}

Trabalhadores expostos a bifenilas policloradas (PCBs) durante a fabricação de capacitores elétricos apresentaram um aumento no risco de morte por câncer de fígado, segundo uma coorte realizada nos EUA entre os anos de 1940 a $1998^{26}$. Trabalhadores expostos ao cloreto de vinila (usado na produção de tubos de plástico) e ao arsênico também apresentaram um risco aumentado de desenvolver câncer de fígado em alguns estudos ${ }^{27-29}$.

\section{Câncer de Laringe}

Alguns estudos têm apontado para uma associação positiva entre câncer de laringe e exposição ocupacional ao pó de madeira em carpintarias e na fabricação de móveis ${ }^{30,31}$. Outros estudos têm observado associação entre exposição ao asbesto e câncer de laringe $\mathrm{e}^{32-34}$.

\section{Câncer de Pulmão e Mesotelioma}

O Agricultural Health Study observou um risco aumentado de câncer de pulmão entre aplicadores de agrotóxicos expostos a organoclorados, a dieldrin ${ }^{35}$ e a carbamatos ${ }^{36}$. O câncer de pulmão também tem sido associado à exposição ocupacional a metais pesados como níquel, cádmio, cromo hexavalente e berílio ${ }^{37,38}$. Um aumento no risco de morte por câncer de pulmão foi observado em um estudo do tipo coorte realizado nos EUA com trabalhadores da indústria de sapatos expostos a solventes como o tolueno ${ }^{39}$. Câncer de pulmão também tem sido associado com a exposição ao asbesto em trabalhadores da indústria de pastilha de freio $^{40} \mathrm{e}$ na fabricação de cimento amianto e tecelagem de tecidos ${ }^{41,42}$. Um estudo do tipo coorte realizado nos EUA com trabalhadores aeroespaciais encontrou um risco aumentado no desenvolvimento de câncer de pulmão quando expostos a óleos minerais ${ }^{43}$.

Um aumento no risco de câncer de pulmão também pode ser observado, segundo alguns estudos, em trabalhadores de minas de carvão ${ }^{44,45}$ e de minas de urânio ${ }^{46}$. Esse aumento no risco de câncer de pulmão também pode ser constatado em trabalhadores expostos ao radônio ${ }^{47,48}$, à fumaça produzida pelo forno de coque $^{41}$, ao cloreto de vinila ${ }^{49} \mathrm{e}$ à combustão de diesel ${ }^{50}$.

A sílica cristalina respirável tem sido associada, em alguns estudos, ao câncer de pulmão em trabalhadores da indústria da construção civil ${ }^{51,52}$, pedreiros ${ }^{53}$ e metalúrgicos ${ }^{54}$.

O mesotelioma maligno de pleura é um tumor de ocorrência rara e tem se mostrado associado à exposição por um longo período ao asbesto, em trabalhadores da construção civil e da indústria têxtil ${ }^{42,55,56}$.
Cânceres Hematológicos (Leucemia,Linfoma e Mieloma Múltiplo)

Quanto à leucemia, a Agricultural Health Study observou um aumento no risco de sua ocorrência em aplicadores de agrotóxicos expostos a organoclorados, principalmente aldrin, clordane, dieldrin e toxafeno ${ }^{35}$. Um aumento no risco de leucemia também tem sido observado entre trabalhadores da indústria de borracha ${ }^{57}$. Novos estudos tem reportado um aumento no risco de leucemia e mieloma múltiplo em trabalhadores da indústria de petróleo expostos a benzeno ${ }^{58-60}$.

Trabalhadores agrícolas expostos a agrotóxicos, principalmente os herbicidas e os fungicidas, têm apresentado um risco aumentado para o desenvolvimento de linfoma não-Hodgkin e mieloma múltiplo ${ }^{20,61-64}$. Alguns estudos têm apontado um aumento no risco de linfoma não-Hodgkin em trabalhadores expostos a solventes como o tolueno e o tricloroetileno ${ }^{65,66}$.

Um estudo do tipo caso-controle de base populacional realizado na Alemanha identificou algumas ocupações associadas ao desenvolvimento de linfoma não-Hodgkin, como: engenheiros, cozinheiros, garçons, metalúrgicos, eletricistas e trabalhadores na área de eletrônica, médicos, dentistas, veterinários, químicos, processamento de comida e bebida ${ }^{67}$.

\section{Câncer de Pele}

Estudos recentes têm revelado um aumento no risco de desenvolvimento de câncer de pele em trabalhadores expostos por longo período à radiação ultravioleta ${ }^{68-70}$. Trabalhadores agrícolas vêm apresentando risco aumentado em desenvolver câncer de pele não apenas por estarem expostos à luz solar durante seu dia de trabalho, mas por estarem expostos a agrotóxicos arsenicais, que também têm sido associados ao câncer de pele ${ }^{27,71}$. O câncer de pele foi fortemente associado com exposição ao óleo mineral em um estudo realizado com trabalhadores aeroespaciais ${ }^{43}$. Alguns estudos vêm observando uma possível associação entre ocupações específicas e câncer de pele. Um estudo do tipo casocontrole realizado entre bombeiros nos EUA observou um risco elevado em desenvolver câncer de pele ${ }^{72}$. Um estudo observou um aumento no risco de câncer de pele em mulheres empregadas como educadoras, caixas de banco e comissárias de bordo, e outro estudo revelou um aumento no risco de câncer de pele em dentistas, bibliotecários, trabalhadores horticultores ${ }^{73,74}$.

\section{Câncer de Estômago}

O câncer de estômago tem sido associado, segundo alguns estudos, a trabalhadores expostos a bifenilas policloradas (PCBs), a fluidos utilizados no beneficiamento de metais, ao chumbo e ao asbesto $26,33,75,76$. Trabalhadores da indústria da borracha também têm apresentado um risco aumentado no desenvolvimento de câncer de estômago, bem como trabalhadores agrícolas e pintores ${ }^{33,77,78}$. 


\section{Câncer de Cavidade Nasal}

Câncer da cavidade nasal têm sido associado com exposição ocupacional ao formoldeído, ao pó da madeira, ao pó de couro e ao óleo mineral ${ }^{33,79}$.

\section{Câncer de Ovário}

O Agricultural Health Study encontrou um aumento no risco de câncer de ovário entre mulheres aplicadoras de agrotóxicos ${ }^{15}$. Trabalhadoras expostas ao asbesto e enfermeiras também mostraram um risco aumentado para câncer de ovário em alguns estudos ${ }^{80-82}$.

\section{Câncer de Próstata}

Recentes estudos têm conseguido estabelecer uma associação entre exposição a agrotóxicos por trabalhadores agrícolas e câncer de próstata ${ }^{15,63,83-85}$. Um estudo do tipo coorte de trabalhadores expostos a bifenilas policloradas (PCBs) durante a fabricação de capacitores elétricos revelou uma associação positiva com câncer de próstata ${ }^{86}$. Um estudo do tipo casocontrole encontrou uma associação de grande magnitude entre trabalhadores expostos ao cádmio e câncer de próstata ${ }^{87}$.

\section{DISCUSSÃO}

As exposições ocupacionais a substâncias ou misturas químicas de potencial carcinogênico conhecido têm preocupado a comunidade científica e impulsionado pesquisas que buscam avaliar os riscos à Saúde Pública e Ambiental. Entre os inúmeros fatores de risco ambientais para o câncer, argumenta-se que os de origem ocupacional seriam aqueles com mais elevado potencial de controle. Entretanto, apenas 20\% das substâncias químicas em uso no ambiente de trabalho apresentam informações toxicológicas adequadas ${ }^{88}$.

O crescimento do campo da Saúde do Trabalhador explicitou que as situações de risco presentes nos ambientes de trabalho modificavam também o padrão de saúde da população em geral, visto que grande contingente desta é constituído pela própria população de trabalhadores, e também porque o processo de produção pode alterar as condições ambientais, ou melhor, ecológico-sociais, que influenciam a saúde de distintos grupos humanos ${ }^{89}$.

Os estudos de exposição ocupacional têm trazido grandes contribuições para a compreensão da carcinogênese humana. Há muitas exposições adicionais no local de trabalho que são suspeitos cancerígenos que necessitam de avaliação adicional para garantir um ambiente de trabalho seguro. A informação de investigações profissionais também é relevante para a população em geral, porque muitas exposições ocupacionais podem ser encontrados fora do local de trabalho ${ }^{90}$.
Ribeiro e Wünsch Filho ${ }^{91}$ afirmam que a mensuração da exposição a agentes cancerígenos nos ambientes de trabalho é uma tarefa complexa, pois habitualmente configuram-se situações ambientais com múltiplas facetas. O tempo é um componente importante para a mensuração deste contato, pois tanto a data do início da exposição quanto a duração são cruciais à latência e à dose acumulada.

De fato, existem algumas dificuldades com relação à sistematização do risco das substâncias químicas para a ocorrência do câncer. Em primeiro lugar, a informação sobre exposições decorrentes de processos industriais é geralmente escassa, não permitindo uma avaliação de exposições específicas. Além disso, as exposições a agentes bem conhecidos, como o benzeno e o cloreto de vinila, ocorrem em diferentes intensidades em situações ocupacionais distintas. As exposições numa dada atividade ocupacional mudam ao longo do tempo (introdução de novos materiais e/ou processos industriais). As listas de exposições se referem a um pequeno número de investigações sobre riscos de câncer. Finalmente, a maioria dos estudos de exposições associada ao câncer ocupacional foi realizada em países desenvolvidos no passado. Atualmente, os níveis de exposições nos países em desenvolvimento é menor que aqueles presentes nos estudos iniciais?.

Entre as estratégias para a efetivação da Atenção Integral à Saúde do Trabalhador, destaca-se a implementação da Rede Nacional de Atenção Integral à Saúde do Trabalhador, cujo objetivo é integrar a rede de serviços do Sistema Único de Saúde (SUS) voltados à assistência e à vigilância, além da notificação de agravos à saúde relacionados ao trabalho em rede de serviços sentinela ${ }^{92}$. O Ministério da Saúde, por meio da Área Técnica de Saúde do Trabalhador (COSAT) e do Instituto Nacional de Câncer (INCA), está sensível a esta necessidade e propôs, por intermédio dos protocolos, articular o poder público na abordagem do câncer, no que tange aos seus fatores causais relacionados ao trabalho. De fato, no ambiente de trabalho, é possível intervir de forma inequívoca no controle da exposição, seja pelo caráter bem-delimitado da população, seja pelo potencial técnico de monitorar e reduzir estes riscos. Desta forma, adota-se no país a concepção de "níveis seguros" para a exposição ocupacional a maior parte dos cancerígenos, o que conflita com o atual conhecimento científico sobre carcinogênese, que não reconhece limites seguros para a exposição do trabalhador aos agentes cancerígenos ${ }^{91}$.

Para consolidar esta estratégia, desde 2004 são publicadas portarias para fortalecer a notificação das doenças ocupacionais, dentre elas o câncer ocupacional. Em 2004, foi publicada a portaria 777, que dispõe sobre os procedimentos técnicos para a notificação compulsória de agravos à saúde do trabalhador em rede de serviços sentinela específico, no $\mathrm{SUS}^{93}$, regulamentando 
a notificação compulsória de agravos à saúde do trabalhador acidentes e doenças relacionados ao trabalho - em rede dexserviços sentinela específica, criando uma Rede Sentinela de Notificação Compulsória de Acidentes e Doenças Relacionados ao Trabalho constituída por Centros de Referência em Saúde do Trabalhador, hospitais de referência para o atendimento de urgência e emergência e ou atenção de média e alta complexidade, credenciados como sentinela, e serviços de atenção básica e de média complexidade credenciados como sentinelas, por critérios a serem definidos em instrumento próprio. E ainda, estabeleceu-se que a rede sentinela será organizada a partir da porta de entrada no sistema de saúde, estruturada com base nas ações de acolhimento, notificação, atenção integral, envolvendo assistência e vigilância da saúde. Define, ao final e ao cabo, que os procedimentos técnicos de Vigilância em Saúde do Trabalhador deverão estar articulados com aqueles da vigilância ambiental, sanitária e epidemiológica.

$\mathrm{Na}$ sequência, em 2010, foi publicada a portaria 2472, que define as terminologias adotadas em legislação nacional, conforme disposto no Regulamento Sanitário Internacional 2005 (RSI 2005), a relação de doenças, agravos e eventos em saúde pública de notificação compulsória em todo o território nacional e estabelece fluxo, critérios, responsabilidades e atribuições aos profissionais e serviços de saúde ${ }^{94}$. Finalmente, em 2011, foi publicada a portaria 104, que retifica a portaria 2472 , e que é a portaria vigente para o estabelecimento do fluxo de vigilância de todas as doenças de notificação compulsória, incluindo as doenças e agravos em saúde do trabalhador, inclusive o câncer ocupacional. Observa-se, com isso, que o sistema encontra-se mais sensível à vigilância do câncer ocupacional, de forma a estimular ações de prevenção primária e secundária nos serviços de saúde ${ }^{95}$.

A principal estratégia para minimizar os riscos ocupacionais para o câncer, portanto, é reduzir ou eliminar a exposição a agentes classificados como cancerígenos. Contudo, deve-se considerar a relação dinâmica entre a exposição ocupacional e o câncer, tendo em vista as modificações constantes nas características de diversas ocupações e a extinção de algumas destas que cedem lugar à emergência de outras, além da alta carga de produção de substâncias nos processos industriais.

Aponta-se como limitação da presente revisão sistemática o fato de ter restringido sua análise à base de referências bibliográficas MEDLINE/PubMed. Apesar de ser a principal base da área, é possível que alguns artigos não tenham sido incluídos.

\section{CONCLUSÃO}

O câncer é um conjunto diverso e complexo de doenças de etiologia multifatorial. Exposições ocupacionais também atuam sobre essa complexidade e têm efeitos diferenciados sobre a diversidade de subtipos de câncer. $\mathrm{Na}$ atual revisão, foram incluídos os estudos que foram limitados às relações entre exposições ocupacionais específicas e subtipos de câncer específicos, adicionando complexidade aos relatórios atuais existentes. Esta decisão pode subestimar levemente a estimativa total de risco ambiental atribuído, mas não afeta nossas conclusões ${ }^{96}$.

Há, hoje, substanciais evidências científicas para apoiar a associação entre o ambiente e câncer ${ }^{97-99}$. Ligações iniciais entre exposições ambientais e câncer data de por volta de 1761, quando John Hill descobriu a associação entre tabaco e câncer nasal ${ }^{100}$, e de 1775, quando Sir Percival Pott observou uma relação entre o câncer de chaminé de varrição e escrotal ${ }^{101}$. Séculos mais tarde, os investigadores continuam a demonstrar e a quantificar esta relação através de estudos de migrantes, familiares, e de correlação, bem como identificação da variação geográfica na incidência de câncer ${ }^{102-105}$. A partir destes estudos epidemiológicos, as estimativas da proporção de câncer atribuíveis ao ambiente têm sido estudadas.

A estimativa de risco atribuível mais citada vem de John Higginson, que declarou que $80-90 \%$ de todos os cânceres são devidos à exposição a fatores ambientais ${ }^{106}$. Em uma revisão por Boffetta et al. ${ }^{107}$, os autores observam que o termo "ambiente" é frequentemente utilizado em sentido amplo para incluir todos os fatores não-genéticos, e em sentido estrito para incluir apenas ar, água, solo e alimentos poluentes. Boffetta et al. ${ }^{107}$ concluíram que o termo "meio ambiente" deve ser abandonado e substituído, em vez dos termos "nãogenética" e "poluentes". Saracci e Vineis ${ }^{108}$ refutam essa ideia, e insistem em manter o termo "meio ambiente", enquanto incitando os pesquisadores a relatar claramente quais os componentes do ambiente a sua estimativa de risco incluem.

O papel do trabalho é freqüentemente subestimado, particularmente em relação às interações gene-ambiente, devido à baixa sensibilidade das estimativas de exposição ocupacional ${ }^{109}$. Erro de classificação é muitas vezes flutuante, tanto no ambiente de trabalho interno e externo ${ }^{110}$. Desta forma, muitas exposições e seus efeitos correspondentes são medidos em níveis baixos, mas têm uma presença onipresente no ambiente, tornando assim a sua verdadeira contribuição etiológica difícil de interpretar ${ }^{111}$.

A principal estratégia para minimizar os riscos ocupacionais para o câncer, portanto, é reduzir ou eliminar a exposição a agentes classificados como cancerígenos. Contudo, deve-se considerar a relação dinâmica entre a exposição ocupacional e o câncer, tendo em vista as modificações constantes nas características de diversas ocupações e a extinção de algumas destas que cedem lugar à emergência de outras, além da alta carga de produção de substâncias nos processos industriais. 


\section{REFERÊNCIAS}

1. World Health Organization. Detection of health impairment in occupational exposure to health hazards. Geneva; 1975. (WHO technical report series, 571).

2. Mendes R. Conceito de Patologia do Trabalho. In: Mendes R (org.), Patologia do Trabalho. Rio de Janeiro: Atheneu; 2005. p. 47-92.

3. Brasil. Ministério da Saúde. Representação no Brasil da OPAS/OMS Doenças relacionadas ao trabalho: manual de procedimentos para os serviços de saúde. Brasília: Ministério da Saúde; 2001, p. 580.

4. Mendes R, Dias EC. Saúde dos trabalhadores. In: Rouquayrol, MZ, Almeida Filho, N (Eds.). Epidemiologia \& Saúde. Rio de Janeiro: Medsi; 1999. p. 431-456.

5. INTERNATIONAL AGENCY FOR RESEARCH ON CANCER Monographs on the evaluation of carcinogenic risks to humans. Occupational exposures in insecticide application, and some pesticides. Lyon, France; v. 53, p. 115-175, 1991.

6. IARC (1972-2004). IARC Monographs on the Evaluation of Carcinogenic Risks to Humans, Vols 1-80. International Agency for Research on Cancer: Lyon.

7. World Health Organization. An overview of the evidence on environmental and occupational determinants of cancer. Asturias, Spain; 2011 (WHO technical report series, p. 1)

8. World Health Organization. The global occupational health network: prevention of occupational cancer. Geneva; 2006 (WHO technical report series, p.2).

9. Clapp RW, Jaconbs M, Loechler E. Environmental and Occupational Causes of Cancer New Evidence, 2005-2007. Rev Environ Health. 2008;23(1):1-37.

10. Stayner L, Steenland K, Dosemeci M, Hertz-Picciotto I. Attenuation of exposure-response curves in occupational cohort studies at high exposure levels. Scand J Work Environ Health 2003;29(4):317-24.

11. Nurminen M, Karjalainen A. Epidemiologic estimate of the proportion of fatalities related to occupational factors in Finland. Scand J Work Environ Health. 2001;27(3):161-213.

12. Bosetti C, Pira E, LaVecchia C. Bladder cancer risk in painters: a review of the epidemiological evidence, 1989-2004. Cancer Causes Control. 2005; 16:997-1008.

13. Band PR, Nhu DL, MacAurthur AC, Fang R, Gallagher RP. Identification of occupational cancer risks in British Columbia: a population-based case-control study of 1129 cases of bladder cancer. J OccupEnviron Med. 2005;47(8):854-8.

14. Baena AV, Allam MF, Diaz-Molina C, Del Castillo AS, Abdel-Rahman AG, Navajas RF. Urinary bladder cancer and the petroleum industry: a quantitative review. Eur J Cancer Prev. 2006;15:493-7.

15. Alavanja MCR, Sandler DP, Lynch CF, Knott C, Lubin JH, Tarone R, et al.. Cancer incidence in the Agricultural Health Study. Scand J Work Env Hea. 2005;31(Suppl 1):39-45.

16. Lee WJ, Colt JS, Heineman EF, McComb R, Weisenburger DD, Lijinsky $\mathrm{W}$ et al. Agricultural pesticide use and risk of glioma in Nebraska, United States. Occup Environ Med. 2005;62:786-92.

17. Nichols L, Sorahan T. Cancer incidence and cancer mortality in a cohort of UK semiconductor workers, 1970-2002. Occupational Medicine 2005;55:625-30.
18. Clapp RW. Mortality among US employees of a large computer manufacturing company: 1969-2001. Environ Health. 2006;5:30-9.

19. Clapp RW., Jacobs, MM., Loechler EL. Environmental and occupational causes of cancer: new evidence 2005-2007. Rev Environ Health. 2008;23(1):1-37.

20. De Roos AJ, Gao DL, Wernli KJ, Fitzgibbons ED, Ziding F, Astrakianakis $\mathrm{G}$ et al. Colorectal cancer incidence among female textile workers in Shanghai, China: a case-cohort analysis of occupational exposures. Cancer Cause Control. 2005;16(10):1177-88.

21. Ritz B, Zhao Y, Krishnadasan A, Kennedy, N, Morgenstern, H. Estimated effects of hydrazine exposure on cancer incidence and mortality in aerospace workers. Epidemiology. 2006;17(2):154-61.

22. Samanic D, Rusiecki J, Dosemeci M, Lubin J, Hoppin JA, et al. Cancer incidence among pesticide applicators exposed to dicamba in the Agricultural Health Study. Environ Health Persp.2006;114(10):1521-6.

23. Meyer A, Alexandre PC, Chrisman J R, Markowitz SB, Koifman RJ, Koifman S. Esophageal cancer among Brazilian agricultural workers: case-control study based on death certificates. Int J Hyg Environ Health. 2011;214(2):151-5.

24. Wernli KJ, Fitzgibbons ED, Ray RM, Gao DL, Li W, Seixas NS, et al. Occupational risk factors for esophageal and stomach cancers among female textile workers in Shanghai, China. Am J Epidemiol. 2006;163(8):717-25

25. Lynge E, Andersen A, Rylander L, et al. Cancer in persons working in dry cleaning in the Nordic countries. Environ Health Persp. 2006;114(2):213-9.

26. Prince MM, Hein MJ, Ruder AM, et al. Update: cohort mortality study of workers highly exposed to polychlorinated by-phenyls (PCBs) during the manufacture of electrical capacitors, 1940-1998. Environ Health. 2006;5:13-22.

27. Yang M. A current global view of environmental and occupational cancers. J Environ Sci Health C Environ Carcinog Ecotoxicol Rev. 2011;29(3):223-49.

28. Hsieh HI, Chen PC, Wong RH, Du CL, Chang YY, Wang JD, et al. Mortality from liver cancer and leukaemia among polyvinyl chloride workers in Taiwan: an updated study. Occup Environ Med. 2011;68(2):120-5.

29. Fedeli U, Mastrangelo G. Vinyl chloride industry in the courtroom and corporate influences on the scientific literature. Am J Ind Med 2011.

30. Ramroth H, Dietz A, Ahrens W, Becher H. Occupational wood dust exposure and the risk of laryngeal cancer: a population based case-control study in Germany. Am J Ind Med. 2008;51(9):648-55.

31. Paget-Bailly S, Cyr D, Luce D. Occupational exposures and cancer of the larynx-systematic review and meta-analysis. J Occup Environ Med. 2012;54(1):71-84.

32. Pira E, Pelucchi C, Piolatto PG, Negri E, Bilei T, La Vecchia C. Mortality from cancer and other causes in the Balangero cohort of chrysotile asbestos miners. Occup Environ Med. 2009;66(12):805-9.

33. Rushton L, Bagga S, Bevan R, Brown TP, Cherrie JW, Holmes P, et al. Occupation and cancer in Britain. Br J Cancer. 2010; 27;102(9):1428-37.

34. Ramroth H, Ahrens W, Dietz A, Becher H. Occupational asbestos exposure as a risk factor for laryngeal carcinoma in a population-based casecontrol study from Germany. Am J Ind Med. 2011;54(7):510-4. 
35. Purdue MP, Jarvholm B, Bergdahl IA, Hayes RB, Baris D. Occupational exposures and head and neck cancers among Swedish construction workers. Scand J Work Environ Health. 2006;32(4):270-5.

36. Bonner MR, Lee WJ, Sandler DP, Hoppin JA, Dosemeci M, Alavanja MCR. Occupational exposure to carbofuran and the incidence of cancer in the Agricultural Health Study. Environ Health Persp. 2005;113(3):285-9.

37. Beveridge R, Pintos J, Parent ME, Asselin J, Siemiatycki J. Lung cancer risk associated with occupational exposure to nickel, chromium VI, and cadmium in two population-based case-control studies in Montreal. Am J Ind Med. 2010;53(5):476-85.

38. Schubauer-Berigan MK, Deddens JA, Couch JR, Petersen MR. Risk of lung cancer associated with quantitative beryllium exposure metrics within an occupational cohort. Occup Environ Med. 2011;68(5):354-60.

39. Lehman EJ, Hein MJ. Mortality of workers employed in shoe manufacturing: an update. Am J Ind Med. 2006;49:535-46.

40. Azari MR, Nasermoaddeli A, Movahadi M, Mehrabi Y, Hatami H, Soori $\mathrm{H}$, et al. Risk assessment of lung cancer and asbestosis in workers exposed to asbestos fibers in brake shoe factory in Iran. Ind Health. 2010;48(1):38-42.

41. Algranti E, Buschinelli JTP, De Capitani EM. Câncer de pulmão ocupacional. J Bras Pneumol. 2010;36(6):784-94.

42. Loomis D, Dement J, Richardson D, Wolf S. Asbestos fibre dimensions and lung cancer mortality among workers exposed to chrysotile. Occup Environ Med. 2010;67(9):580-4.

43. Zhao Y, Krishnadasan A, Kennedy N, Morgenstern H. Estimated effects of solvents and mineral oils on cancer incidence and mortality in a cohort of aerospace workers. Am J Ind Med. 2005;48(4):249-58.

44. Christian WJ, Huang B, Rinehart J, Hopenhayn C. Exploring geographic variation in lung cancer incidence in Kentucky using a spatial scan statistic: elevated risk in the Appalachian coal-mining region. Public Health Rep. 2011;126(6):789-96.

45. Hosgood HD, Chapman RS, Wei H, He X, Tian L, Liu LZ, et al. Coal mining is associated with lung cancer risk in Xuanwei, China. Am J Ind Med. 2012;55(1):5-10.

46. Rage E, Vacquier B, Blanchardon E, Allodji RS, Marsh JW, Caër-Lorho S, et al. Risk of Lung Cancer Mortality in Relation to Lung Doses among French Uranium Miners: Follow-Up 1956-1999. Radiat Res. 2012;177(3):288-97.

47. Vacquier B, Rage E, Leuraud K, Caër-Lorho S, Houot J, Acker A, et al. The influence of multiple types of occupational exposure to radon, gamma rays and long-lived radionuclides on mortality risk in the French "post-55" sub-cohort of uranium miners: 1956-1999. Radiat Res. 2011;176(6):796-806.

48. Sethi TK, El-Ghamry MN, Kloecker GH. Radon and lung cancer. Clin Adv Hematol Oncol. 2012;10(3):157-64.

49. Gennaro V, Ceppi M, Crosignani P, Montanaro F. Reanalysis of updated mortality among vinyl and polyvinyl chloride workers: Confirmation of historical evidence and new findings. BMC Public Health. 2008;22(8):21.

50. Luce D, Stücker I; ICARE Study Group. Investigation of occupational and environmental causes of respiratory cancers (ICARE): a multicenter, population-based case-control study in France. BMC Public Health. 2011;14(11):928.

51. Carneiro AP, Santos MA, Maia PV, Barreto SM. Câncer de pulmão em trabalhadores expostos à sílica. J Pneumol. 2002;28(4):233-6.
52. Vida S, Pintos J, Parent ME, Lavoué J, Siemiatycki J. Occupational exposure to silica and lung cancer: pooled analysis of two casecontrol studies in Montreal, Canada. Cancer Epidemiol Biomarkers Prev. 2010;19(6):1602-11.

53. Consonni D, De Matteis S, Pesatori AC, Cattaneo A, Cavallo DM, Lubin $\mathrm{JH}$, et al. Increased lung cancer risk among bricklayers in an Italian population-based case-control study. Am J Ind Med. 2012;48:249-58.

54. Wünsch-Filho V, Moncau JE, Mirabelli D, Boffetta P. Occupational risk factors of lung cancer in Sao Paulo, Brazil. Scand J Work Environ Health. 1998;24:118-24

55. Li P, Deng SS, Wang JB, Iwata A, Qiao YL, Dai XB, et al. Occupational and environmental cancer incidence and mortality in China. Occup Med (Lond). 2012;12.

56. Siesling S, Zwan JM, Izarzugaza I, Foschi R, Ricardi Uet al; on behalf of the RARECARE working group. Rare thoracic cancers, including peritoneum mesothelioma. Eur J Cancer. 2012;9.

57. Alder N, Fenty J, Warren F, Sutton AJ, Rushton L, Jones DR, et al. Meta-analysis of mortality and cancer incidence among workers in the synthetic rubber-producing industry. Am JEpidemi. 2006;164(5):405-20.

58. Sorahan T, Kinlen LJ, Doll R. Cancer risks in a historical UK cohort of benzene exposed workers. Occup Environ Med. 2005;62:231-6.

59. Glass DC, Gray CN, Jolley DJ, Gibbons C, Sim MR.. The health watch case-control study of leukemia and benzene. An NY Acad Sci. 2006;1076:80-9.

60. Khalade RA, Jaakkola SM, Pukkala E, Jaakkola JJK. Expousure to benzene at work and the risk of leukemia: a systematic review and metaanalysis. Environ Health. 2010, 9:31.

61. Mills PK, Yang R, Riordan D. Lymphohematopoietic cancers in the United Farm Workers of America (UFW), 1988-2001. Cancer Cause Control. 2005;16:823-30.

62. Rafnsson V. Risk of non-Hodgkin's lymphoma and exposure to hexachlorocyclohexane, a nested case-control study. Europ J Cancer. 2006;42:2781-5.

63. Lynch SM, Rusiecki JA, Blair A, Dosemeci M, Lubin J, Sandler $\mathrm{D}$, et al. Cancer incidence among pesticide applicators exposed to cyanazine in the Agricultural Health Study. Environ Health Persp. 2006;114(8):1248-52.

64. Burns C, Bodner K, Swaen G, Collins J, Beard K, Lee M Cancer incidence of 2,4-D production workers. Int J Environ Res Public Health. 2011;8(9):3579-90.

65. Fritschi L, Benke G, Hues AM, Kricker A, Turner J, Vajdic CM, et al. Risk of non-Hodgkin's lymphoma associated with occupational exposure to solvents, metals, organic dusts and PCBs (Australia). Cancer Cause Control. 2005;16:599-607.

66. Purdue MP, Bakke B, Stewart P, De Roos AJ, Schenk M, Lynch CF, et al. A case-control study of occupational exposure to trichloroethylene and non-Hodgkin lymphoma. Environ Health Persp. 2011;119(2).

67. Mester B, Nieters A, Deeg E, Elsner G, Becker N, Seidler A. Occupation and malignant lymphoma: a population based case control study in Germany. Occup Environ Med. 2006;63:17-26.

68. Pukkala E, Martinsen JI, Lynge E, Gunnarsdottir HK, Sparén P, Tryggvadottir L, et al. Occupation and cancer - follow-up of 15 million people in five Nordic countries. Acta Oncol. 2009;48(5):646-790. 
69. Parkin DM, Mesher D, Sasieni P. Cancers attributable to solar (ultraviolet) radiation exposure in the UK in 2010. $\mathrm{Br}$ J Cancer. 2011;6:105(Suppl 2).

70. Vernez D, Milon A, Vuilleumier L, Bulliard JL. Anatomical exposure patterns of skin to sunlight: relative contributions of direct, diffuse and reflected ultraviolet radiation. Br J Dermatol. 2012;22.

71. Melkonian S, Argos M, Pierce BL, Chen Y, Islam T, Ahmed A, et al. A prospective study of the synergistic effects of arsenic exposure and smoking, sun exposure, fertilizer use, and pesticide use on risk of premalignant skin lesions in Bangladeshi men. Am J Epidemiol. 2011;173(2):183-91.

72. Bates MN. Registry-based case-control study of cancer in California firefighters. Am J Ind Med. 2007;50:39-44.

73. Tokumaru O, Haruki K, Bascal K, Katagiri T, Yamamoto T, Sakurai Y. Incidence of cancer among female flight attendants: a metaanalysis. Journal of Travel Medicine 2006;13(3):127-32.

74. Perez-Gomez B, Aragones N, Gustavsson P, Plato N, Lopez-Abente G, Pollan M.. Cutaneous melanoma in Swedish women: occupational risks by anatomic site. Am J Ind Med. 2005;48(4):270-81.

75. Bunderson-Schelvan M, Pfau JC, Crouch R, Holian A. Nonpulmonary outcomes of asbestos exposure. J Toxicol Environ Health B Crit Rev. 2011;14(1-4):122-52

76. Santibañez M, Alguacil J, de la Hera MG, Navarrete-Muñoz EM, Llorca $\mathrm{J}$, et al; for the PANESOES Study Group. Occupational exposures and risk of stomach cancer by histological type. Occup Environ Med. 2012;69(4):268-75.

77. Chen R, Seaton A. A meta-analysis of painting exposure and cancer mortality. Cancer Detect Prev. 1998;22(6):533-9.

78. Blair A, Freeman LB. Epidemiologic studies in agricultural populations: observations and future directions. J Agromedicine 2009;14(2):125-31.

79. Arias Bahia SH, Echenique Mattos I, Koifman S. Cancer and woodrelated occupational exposure in the Amazon region of Brazil. Environ Res. 2005;99(1):132-40.

80. Lie JS, Andersen A, Kjaerheim K. Cancer risk among 43,000 Norwegian nurses. Scand J Work Environ Health. 2007;33(1):66-73.

81. Camargo MC, Stayner LT, Straif K, Reina M, Al-Alem U, Demers PA, et al. Occupational exposure to asbestos and ovarian cancer: a metaanalysis. Environ Health Perspect. 2011;119(9):1211-7.

82. Reid A, de Klerk N, Musk AW. Does exposure to asbestos cause ovarian cancer? A systematic literature review and meta-analysis. Cancer Epidemiol Biomarkers Prev. 2011;20(7):1287-95.

83. Mahajan R, Blair A, Lynch CF, Schroeder P, Hoppin JA, Sandler DP, et al. Fonofos exposure and cancer incidence in the Agricultural Health Study. Environ Health Persp. 2006;114(12):1838-42.

84. Mahajan R, Bonner MR, Hoppin JA, Alavanja MC. Phorate exposure and incidence of cancer in the Agricultural Health Study. Environ Health Persp. 2006;114(8):1205-9.

85. Bassil KL, Vakil C, Sanborn M, Cole DC, Kaur JS, Kerr KJ. Cancer health effects of pesticides: systematic review. Can Fam Physician. 2007;53(10):1704-11.

86. Prince MM, Ruder AM, Hein MJ, Ruder AM, Waters MA, Laber PA, et al. Mortality and exposure response among 14,458 electrical capacitor manufacturing workers exposed to polychlorinated biphenyls (PCBs). Environ Health Persp. 2006;114(10):1508-14.
87. Vinceti M, Venturelli M, Sighinolfi C, Trerotoli P, Bonvicini F, Ferrari A, et al. Case-control study of toenail cadmium and prostate cancer risk in Italy. Sci Total Environ. 2007;373(1):77-81.

88. Wünsch Filho V, Koifman S. Tumores malignos relacionados com o trabalho. In: Mendes R (org.). Patologia do Trabalho; 2005; Rio de Janeiro: Atheneu; 2005. p. 47-92.

89. Tambellini AT, Camara VM. A temática saúde e ambiente no processo de desenvolvimento do campo da saúde coletiva: aspectos históricos, conceituais e metodológicos. Cienc Saúde Colet. 1998;3(2):47-59.

90. Blair A, Marrett L, Beane Freeman. Occupational cancer in developed countries Environmental Health 2011;10(Suppl 1):S9.

91. Ribeiro FSN, Wünsch Filho V. Avaliação retrospectiva da exposição ocupacional a cancerígenos: abordagem epidemiológica e aplicação em vigilância em saúde. Cad Saúde Pública. 2004;20(4):881-90.

92. Brasil. Ministério da Saúde. Secretaria de Atenção à Saúde. Departamento de Ações Programáticas Estratégicas. Câncer relacionado ao trabalho: leucemia mielóide aguda - síndrome mielodisplásica decorrente da exposição ao benzeno/Ministério da Saúde, Secretaria de Atenção à Saúde, Departamento de Ações Programáticas Estratégicas. Brasília: Editora do Ministério da Saúde, 2006.

93. Brasil. Ministério da Saúde. Portaria GM/MS 777/2004. Dispõe sobre os procedimentos técnicos para a notificação compulsória de agravos à saúde do trabalhador em rede de serviços sentinela específica, no Sistema Único de Saúde - SUS. Brasília, 2004.

94. Brasil. Ministério da Saúde. Portaria GM/MS 2472/2010. Define as terminologias adotadas em legislação nacional, conforme disposto no Regulamento Sanitário Internacional 2005 (RSI 2005), a relação de doenças, agravos e eventos em saúde pública de notificação compulsória em todo o território nacional e estabelecer fluxo, critérios, responsabilidades e atribuições aos profissionais e serviços de saúde. Brasília, 2010.

95. Brasil. Ministério da Saúde. Portaria GM/MS 104/2011. Define as terminologias adotadas em legislação nacional, conforme disposto no Regulamento Sanitário Internacional 2005 (RSI 2005), a relação de doenças, agravos e eventos em saúde pública de notificação compulsória em todo o território nacional e estabelecer fluxo, critérios, responsabilidades e atribuições aos profissionais e serviços de saúde. Brasília, 2011.

96. ATSDR, Agency for Toxic Substances \& Disease Registry, 2010. What you Need to Know. What You Can Do. What causes cancer? Atlanta, Georgia. Available from: http://www.atsdr.cdc.gov/risk/cancer/cancercauses.html

97. Tomatis L, Bartsch H. The contribution of experimental studies to risk assessment of carcinogenic agents in humans. Exp Pathol. 1990;40(4):251-66.

98. Boffetta P. Human cancer from environmental pollutants: the epidemiological evidence. Mutat Res Genet Toxicol Environ Mutagen. 2006;608(2):157-62.

99. Clapp RW, Howe GK, Jacobs MM. Environmental and occupational causes of cancer: a call to act on what we know. Biomed Pharmacother. 2007;61(10):631-9.

100.Hill, J. In: Baldwin, R., Jackson, J. (Eds.), Cautions Against the Immoderate use of Snuff. London, UK; 1761.

101. Pott P., 1775. Chirurgical observations relative to the cataract, the polypus of the nose, the cancer of the scrotum, the different kinds of ruptures, and the mortification of the toes and feet. (Reprinted in Natl Cancer Inst Monogr. 1963;10:7-13). 
102. Higginson J, Muir CS. Determination of the importance of environmental factors in human cancer: the role of epidemiology. Bull Cancer. 1977;64(3)365-84.

103. Parkin DM. Studies of cancer in migrant populations: methods and interpretation. Rev. Epidemiol. Sante Publique. 1992;40(6):410-24.

104. Verkasalo PK, Kaprio J, Koskenvuo M, Pukkala E. Genetic predisposition, environment and cancer incidence: a nationwide twin study in Finland, 1976-1995. Int J Cancer. 1999;83(6):743-9.

105. Lichtenstein P, Holm NV, Verkasalo PK, Iliadou A, Kaprio J, Koskenvuo $\mathrm{M}$, et al. Environmental and heritable factors in the causation of canceranalyses of cohorts of twins from Sweden, Denmark, and Finland. N. Engl J Med. 2000;343(2):78-85.

106. Higginson J. Environment and cancer. Practitioner. 1967;198(187):621-30.
107. Boffetta P, McLaughlin JK, la Vecchia C, Autier P, Boyle P. 'Environment' in cancer causation and etiological fraction: limitations and ambiguities. Carcinogenesis. 2007;28(5):913-5.

108. Saracci R, Vineis P. Disease proportions attributable to environment. Environ Health. 2007;6:38.

109. Brunekreef, B. Environmental epidemiology and risk assessment. Toxicol Lett. 2008;180(2):118-22.

110. Rappaport, SM, Smith MT. Epidemiology. Environment and disease risks. Science. 2010;330(6003):460-1.

111. Wild CP. Environmental exposure measurement in cancer epidemiology. Mutagenesis. 2009;24(2):117-25.

Recebido em: 16/05/2012 Aprovado: 10/01/2013 